\title{
INFLUENCIA DE LOS ADITIVOS ORGÁNICOS NATURALES EN LA RESISTENCIA A LA CRISTALIZACIÓN DE SALES EN MORTEROS TRADICIONALES DE CAL CON DISTINTO ÁRIDO. ANÁLISIS DEL CASO: SITIO ARQUUEOLÓGICO LA BLANCA, PETÉN, GUATEMALA ${ }^{(*)}$
}

\author{
INFLUENCE OF NATURAL ORGANIC ADDITIVES ON THE RESISTANCE TO SALT CRYSTALLIZATION IN TRADITIONAL \\ LIME MORTARS WITH DIFFERENT ARID. CASE ANALYSIS: LA BLANCA ARCHAEOLOGICAL SITE, PETÉN, GUATEMALA
}

\author{
FRANCISCA LORENZO MORA ${ }^{(\star \star)} Y$ BEGONA CARRASCOSA MOLINER ${ }^{[\star \star *]}$ \\ (D) https://orcid.org/0000-0002-0683-1329 \\ lorenzomorafran@gmail.com \\ Universitat Politècnica de València (España)
}

Fecha de recepción: 14 de diciembre de 2018

Fecha de aprobación: 19 de marzo de 2019

\begin{abstract}
RESUMEN
La Blanca es un asentamiento maya del periodo Clásico Terminal, el cual se ubica en la selva del Petén guatemalteco. En las imponentes estancias que componen su acrópolis, se han hallado gran parte de los estucos que sirvieron de revestimiento interior, incluso con huellas de color. Desde que estas estancias fueron excavadas, los estucos se han visto afectados por el agua que asciende por capilaridad y la cristalización de sales, tanto en la superficie como en el soporte pétreo, que han causado graves patologías, como laminaciones y la desintegración del material. Por ello, se están realizando estudios de laboratorio con distintos morteros de cal que pueden ser empleados en la restauración con total garantía. En este trabajo, se presentan los resultados comparativos de estabilidad frente a los ciclos de cristalización de sales de dos tipos de morteros tradicionales de cal, y se evalúa la influencia que los aditivos orgánicos suponen en la mejora de la resistencia.
\end{abstract}

\section{PALABRAS CLAVE}

Estuco; conservación; cristalización

\begin{abstract}
La Blanca is a Terminal Classic period Mayan settlement, located in the Petén jungle of Guatemala. In the imposing rooms that form the Acropolis, there were found much of the stucco which formed the inner lining, even with traces of colour. Since these rooms were excavated, the stuccos have been affected by the water that rises by capillarity and the salts crystallization, both on the surface and in the stone support, which has caused serious pathologies such as laminations and material disintegration. For this reason, laboratory studies are being carried out with different lime mortars that can be used in the restoration with full guarantee. In this paper, the comparative results of stability against salts crystallization cycles on two kind of traditional lime mortars are presented, and the influence that organic additives have on the improvement of resistance is evaluated.
\end{abstract}

\section{KEYWORDS}

Stucco; conservation; crystallization

\footnotetext{
${ }^{*}$ ) El presente trabajo forma parte de la investigación para la tesis doctoral Los estucos mayas. Estudio físico-mecánico de variantes técnicas y su aplicación en la conservación y restauración en el sitio arqueológico La Blanca, Petén, Guatemala; inscrito en el programa de doctorado Ciencia y Restauración por la Universitat Politècnica de València (UPV).

(**) Licenciada en Bellas Artes (2008) y Máster en Conservación y Restauración de Bienes Culturales (2010) por la UPV. Actualmente cursa estudios de doctorado en el programa Ciencia y Restauración del Patrimonio Histórico-Artístico, cuya tesis se centra en el estudio de materiales y técnicas de manufactura de los estucos prehispánicos y su aplicación en intervenciones de conservación. Desde el 2007, participa en el Proyecto arqueológico La Blanca, realizando labores de conservación de los estucos, e investigación práctica in situ con materiales del entorno.

${ }^{(* * *}$ ) Doctora en Bellas Artes (1995, UPV). Desde 1992 es profesora titular del Departamento de Conservación y Restauración de Bienes Culturales de la Facultad de Bellas Artes en la UPV. Ha sido Directora del Museo de Prehistoria y del Servicio de Investigación Prehistórica de la Diputación de Valencia (1996-2000). Ha participado y dirigido diversos proyectos de I+D+i (en España, Guatemala y Ecuador). Además, es miembro del Consejo Rector del Instituto de Conservación y Restauración de La Conselleria de Cultura, Educación y Ciencia de Valencia y del Consejo Científico del Máster de Conservación y Restauración de Bienes Culturales de la UPV.
} 
devenir Vol. 6, N¹2, JULIO - DICIEMBRE 2019, PP. 11-26 - Estudios I ISSN 2312-7562 | E-ISSN 2616-4949

UNIVERSIDAD NACIONAL DE INGENIERÍA, LIMA

doi: https://doi.org/10.21754/devenir.v6i12.741

\section{Introducción}

El estuco fue el material por excelencia empleado por la cultura maya, el cual jugó un papel fundamental en la construcción de las ciudades. La monumental y característica arquitectura maya, hoy convertida en vestigios, no se concibe sin el estuco; en primer lugar, porque la conservación de las construcciones en un clima tropical hubiera estado muy limitada, y en segundo lugar, porque el estuco y la pintura fueron los transmisores de aquellas historias, hechos o representaciones con las que sus gobernantes hicieron a los edificios hablar. Para el acabado de las construcciones, se empleó en gran abundancia esta pasta de cal, que se aplicó tanto como revestimiento interior y exterior de los edificios como para modelar grandes relieves decorativos en los exteriores de sus fachadas.

La técnica adquirida en su preparación, la abundancia de compuestos calizos como materia prima y las óptimas propiedades plásticas para recubrir suelos, muros o relieves ornamentales sobre los que aplicar el color lo convirtieron en un material imprescindible para la construcción de las ciudades. Los mayas aprovecharon la abundancia de recursos naturales a su disposición para elaborar sus estucos, mejorando sus propiedades con respecto de las necesidades de un clima tan agresivo.

Si bien en el área maya las cales que se emplearon para la elaboración de los estucos fueron fundamentalmente aéreas, procedentes de calizas muy puras o grasas (Schreiner, 2002, p. 15), se han reportado casos en los que los estucos presentan compuestos volcánicos o meteóricos (Villaseñor y Barba, 2011; Barba, 2013; Villaseñor y Graham, 2010; Magaloni, 1995) que bien pudieron ser mezclados intencionadamente para incrementar la dureza y resistencia de los estucos una vez carbonatados. La piedra caliza con la que elaboraron la cal fue transformada en cal viva $(\mathrm{CaO})$ mediante su calcinación en grandes piras de leña de alto contenido de humedad (Schreiner, 2002, p. 32). Esta piedra caliza local hoy en día aún es seleccionada por caleros de la región de Petén, quienes emplean distintos términos para referirse a ellas según sus propiedades. Sascab tunich es una piedra suave y porosa, similar a tzaal, que también es calcinada en Petén para elaborar la cal, al igual que sakeel b'aach, o piedra blanca, aunque esta última es algo más cristalina. Por el contrario, términos como toc tunich, taman tunich, piedra de cerro o piedra de fuego (Schreiner, 2002, p. 53), hacen referencia a piedras dolomíticas ${ }^{1}$ y silíceas ${ }^{2}$ muy poco porosas y, por ello, no adecuadas para la obtención de cal.

Como material de carga para la formación del mortero, los mayas emplearon especialmente un árido: el sascab ${ }^{3}$. A su empleo como componente de las pastas de cal, se hace referencia en la obra de fray Diego de Landa (2002) en un fragmento que dice:

Que están los edificios muy cerca unos de otros y que son templos y que la razón de haber tantos es por mudarse las poblaciones muchas veces; y que en cada pueblo labraban un templo por el gran aparejo que hay de piedra y cal y cierta tierra blanca excelente para edificios [...]. (p. 12)

La hidratación o apagado de la cal difiere según las fuentes etnográficas, de manera que se encuentran diversos sistemas que pudieron haberse dado en tiempos prehispánicos. Factores geográficos, económicos, sociales o técnicos constituyen el abanico de posibili-

\footnotetext{
1. Rocas dolomíticas: Pertenecen al grupo de las rocas carbonatadas y su composición es principalmente calcita $\left(\mathrm{CaCO}_{3}\right)$ y carbonato de magnesio $\left(\mathrm{MgCa}\left(\mathrm{CaCO}_{3}\right)_{2}\right)$. En función del porcentaje de calcita y dolomita que presentan las rocas, reciben distintos nombres: calizas, calizas dolomíticas, dolomías calcáreas y dolomías (Moreno et al., s. f., p. 5).

2. Rocas silíceas: Pertenecen al grupo de las rocas sedimentarias y se caracterizan por su alto contenido en sílice $\left(\mathrm{SiO}_{2}\right)$.

3. Proviene de la palabra maya sah cab, que significa «tierra blanca» y se identifica como carbonatos no consolidados, sobre los que se superponen capas de sedimentos calizos, que existen como afloramientos en gran parte de las tierras bajas.
} 
dades técnicas y las diferencias de ejecución en las distintas fases de elaboración de los estucos (Villaseñor y Barba, 2012, pp. 11-41). El apagado por pudrición a cielo abierto es un método empleado todavía a día de hoy en la región de Petén. Para este método, la cal viva recién calcinada se deja en el sitio para que absorba la humedad ambiental, del suelo o de la lluvia. Al principio, esta cal se expande y se convierte en un polvo blanco y brillante de hidróxido cálcico. Después de un tiempo, se empieza a formar una costra oscura en la superficie, aunque el interior permanece intacto. La cal viva puede apagarse también, dejándose expuesta mezclada junto al árido correspondiente (Muñoz, 2006, p. 60).

Para este método de apagado por exposición ambiental, las piedras de cal viva recién calcinadas no necesitan ser transportadas ni preparadas para su apagado; no se requiere de la construcción de estructuras ni tampoco es necesario el transporte de agua hasta el lugar, por lo que supone un sistema rápido y cómodo, si se tiene en cuenta la elevada humedad relativa del área, así como las frecuentes lluvias.

Para mejorar las propiedades de los estucos, los artesanos mayas integraron un tercer componente: los exudados vegetales procedentes de cortezas de algunos árboles. El procedimiento de macerado en agua, mediante el cual estas sustancias eran extraídas, se encuentra relatado en documentos coloniales:

Lo alto era de terrado encalado muy fuerte, como allá se hace con cierta agua de corteza de un árbol [...] Otros árboles de cuyas cortezas hacen los indios cubillos para sacar agua para sí, y otros de que hacen las sogas, y otros de cuyas cortezas majadas hacen un caldo para bruñir con él los encalados, y los hace muy fuertes [...]. (De Landa, 2002, pp. 95-112)

Además, sus efectos han sido ampliamente estudiados, por lo que se concluye que estas gomas tienen un efecto en las propiedades de la cal, el cual estabiliza la estructura durante la carbonatación (Littman, 1960; Magaloni, 2001).

Cada fragmento de este minucioso proceso, desde la selección de la materia prima hasta la aplicación del estuco, fue llevado a cabo con prolijo trabajo, gracias a lo cual gran parte del estuco ha conseguido sobrevivir hasta nuestros días, a pesar de las duras condiciones ambientales y al periodo de sepultura sobrevenido desde el colapso de la arquitectura que lo sustenta. Este es el caso del sitio arqueológico La Blanca, un asentamiento que tuvo su periodo de esplendor en el Clásico Terminal (850-1000 n. e.) y del cual sus estancias quedaron totalmente colapsadas tras el abandono de la ciudad (ver Figura 1). Las ruinas de La Blanca se ubican en el departamento de Petén, en el corazón de las tierras bajas mayas, en una planicie aluvial de formación cuaternaria (MAGA, 2001, p. 83) que se corresponde con el valle del río Mopán. El clima se caracteriza por ser cálido, con elevadas precipitaciones. Las temperaturas son altas durante todo el año (especialmente en los meses de marzo a mayo), con temperaturas diurnas de $29^{\circ} \mathrm{C}-32^{\circ} \mathrm{C}$ y nocturnas de $20^{\circ} \mathrm{C}-24^{\circ} \mathrm{C}$, razón por la que se produce mayor contraste térmico entre el día y la noche que entre las distintas estaciones (Grübe, 2000, p. 22).

La arquitectura maya destaca por sus técnicas constructivas monumentales y sofisticadas, sobre todo en los periodos de máximo esplendor (Clásico, 250 a. n. e. - 1000 n. e.). Se caracteriza por el empleo de la superposición constructiva, mediante la cual, a menudo, los edificios eran clausurados y soterrados bajo las nuevas construcciones. Gruesos muros de sillares con un relleno de mampostería formaban y definían los espacios de los edificios, que culminaban con imponentes cresterías, conseguidas gracias al empleo de la bóveda por aproximación ${ }^{4}$. Las fachadas de los edificios, ri- 


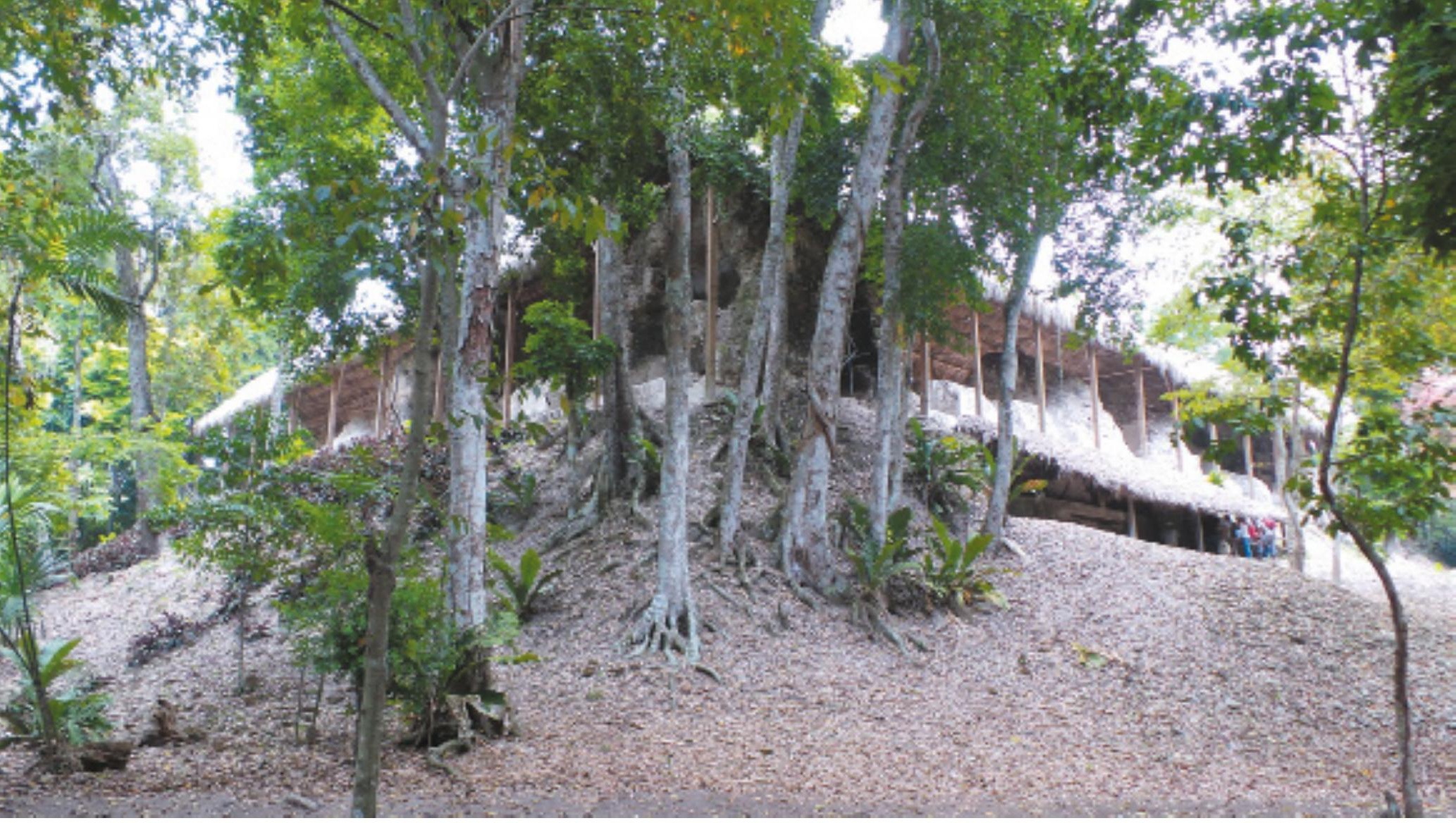

Figura 1. Esquina noroeste de la Acrópolis de La Blanca. Archivo fotográfico de las autoras, 2018. camente decoradas en estuco modelado y pintado, guardan una simetría y ritmo compositivo, con lo que otorgan un carácter único a sus ciudades.

Desde 2004, el proyecto La Blanca investiga el lugar y, hasta la fecha, se ha sacado a la luz gran parte de sus construcciones más señaladas. Al liberar las estancias que componen la acrópolis (ver Figura 2), se ha hallado buena parte del estuco de recubrimiento interior, en el que todavía se pueden encontrar estratos de color e interesantes grafitos incisos.

Durante este periodo de sepultura, estos estucos han sufrido daños mecánicos derivados en gran parte del asentamiento arquitectónico, lo que ha ocasionado patologías como roturas, fisuras y desprendimientos. Otros daños muy presentes se derivan del crecimiento de raíces entre el estuco y el soporte pétreo, lo que desencadena el debilitamiento material, fracturas, fisuras y la separación entre ambos estratos (ver Figura 3). A pesar de ello, el medio de sepultura proporcionó un equilibrio a estos materiales y llegó a alcanzar con el tiempo cierta estabilidad que favoreció a su conservación.

Desde la excavación, los nuevos agentes ambientales combinados afectan a los estucos. La excavación implica un fuerte trauma para estos materiales, ya que supone un cambio repentino en las condiciones de humedad, temperatura, luz y oxígeno en las que se encontraban estables; y es a partir de aquí cuando estos materiales comienzan a manifestar las patologías más severas, en su intento de equilibrarse con las nuevas condiciones, y a pesar de las medidas de protección y techado de las ruinas (ver Figura 4).

El agua es uno de los agentes de deterioro más importantes, ya que genera por sí sola, o en combinación con otros factores, una gran cantidad de daños en los elementos calizos y puede desencadenar reacciones químicas y ataques biológicos. Debido a la naturaleza polar del agua, se ve atraída por las superficies hidrófilas y es absorbida por los pequeños poros o grietas de la superficie. En este entorno selvático, debido a la presencia inevitable del agua, el contacto con los materiales se produce a través de filtraciones de lluvia, por la absorción de la propia humedad ambiental o por la condensación producida por la diferencia de temperatura entre el material y la humedad ambiental.

El proceso de deterioro que causa más daños en los materiales constructivos es la saturación de humedad, que es absorbida por el fenómeno físico de capilaridad, un proceso 


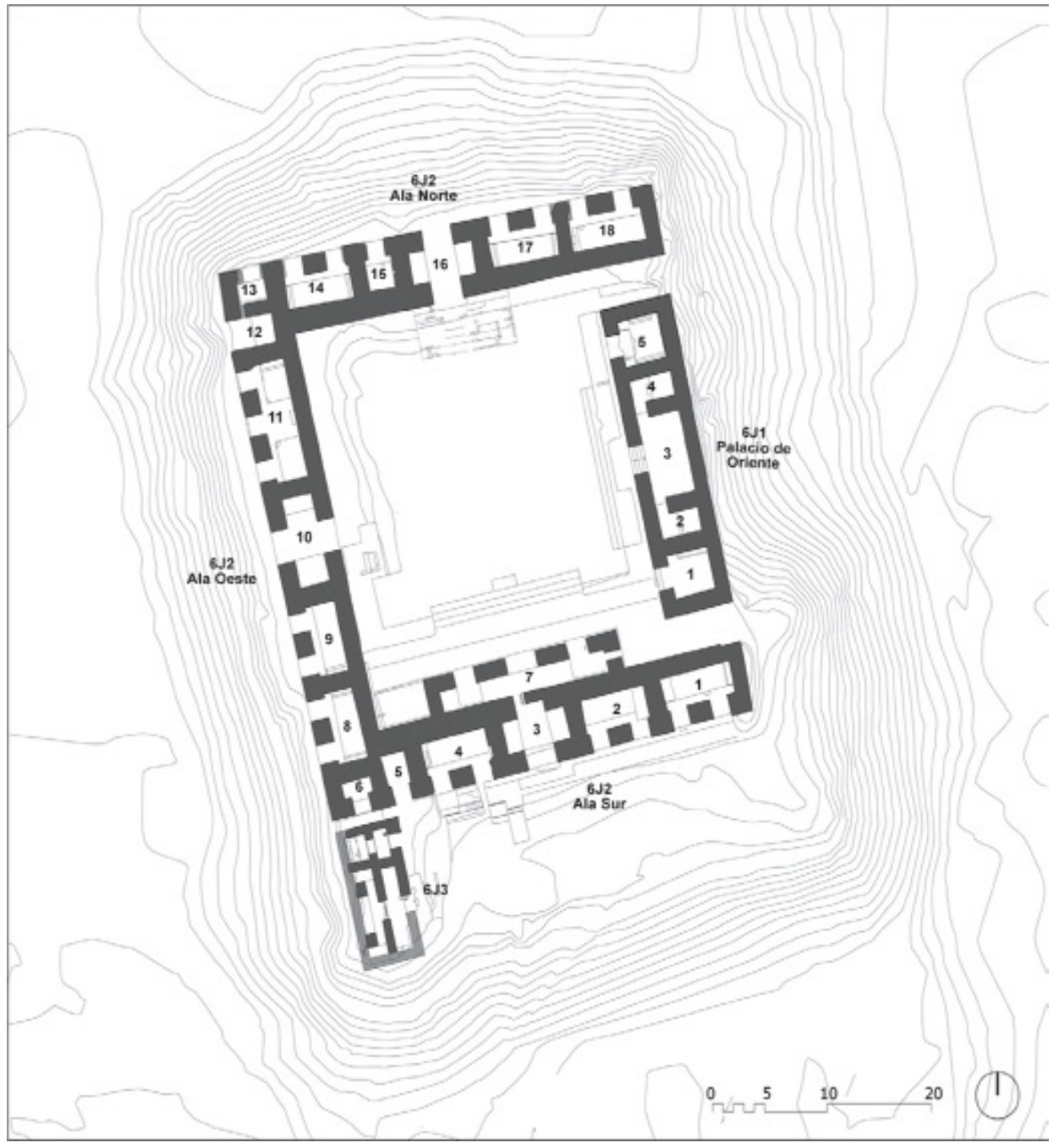

Figura 2. Planta de la Acrópolis de La Blanca. Archivo planimétrico de las autoras, 2018.

que implica el ascenso y descenso de un líquido en el interior de la red porosa, y se debe a la presencia de fuerzas interfase entre sustancias sólidas, líquidas y gaseosas en contacto, denominadas tensión superficial (Kröner et al., 2010). En materiales porosos, cuando la distancia entre las paredes de los poros es del orden de magnitud del micrón $(\mu \mathrm{m})$, la atracción hacia las paredes prevalece sobre la atracción entre moléculas de agua, lo que permite que el agua ascienda hasta alturas considerables. En el caso de muros constructivos, al estar en contacto con el suelo, el agua asciende por la red porosa hasta establecer un equilibrio, cuando la cantidad de agua evaporada a través de la superficie compense el consumo de agua desde el suelo (Torraca, 2009, p. 82). El comportamiento del agua a través de la red porosa de los materiales, y en función de las condiciones ambientales, determina el deterioro de dicho material, dado que viaja acompañada de sustancias disueltas, como las sales solubles y otros compuestos, que pueden afectar gravemente e incluso destruir los enlucidos por la cristalización ${ }^{5}$ repetida de sales y las reacciones químicas desencadenadas entre otros compuestos y los materiales pétreos.

5. Es el proceso por el cual las sales solubles disueltas en el agua ambiental forman una red cristalina pasando del estado líquido al sólido al evaporarse el disolvente.

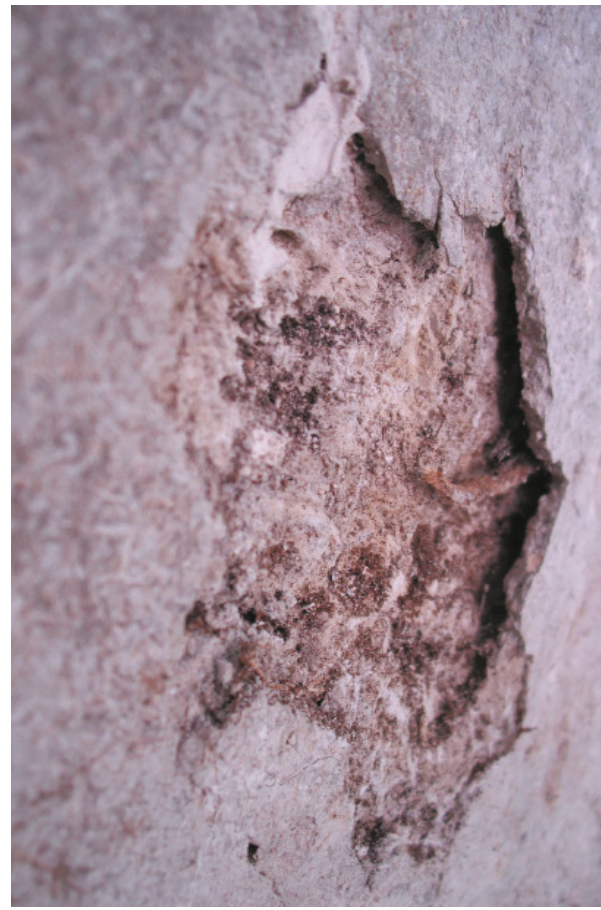

Figura 3. Crecimiento de raíces entre el estuco y el soporte pétreo. Archivo fotográfico de las autoras, 2018.

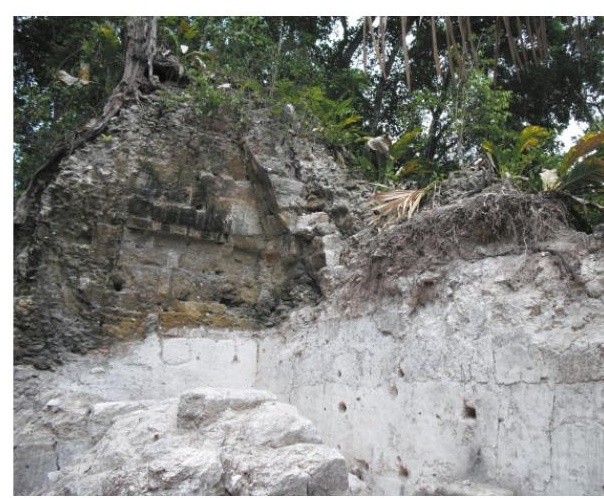

Figura 4. Cuarto 6J2-11 recién excavado y expuesto a las nuevas condiciones ambientales. Archivo fotográfico de las autoras, 2018. 
dratado (yeso) $\left(\mathrm{CaSO}_{4} \cdot 2 \mathrm{H}_{2}\right.$ ) y el sulfato de magnesio $\left(\mathrm{MgSO}_{4}\right)$. También, es común encontrar cloruro de sodio ( $\mathrm{NaCl})$, nitrato de sodio $\left(\mathrm{NaNO}_{3}\right)$ y nitrato de potasio $\left(\mathrm{KNO}_{3}\right)$. La cristalización de sales producida en la superficie de los revestimientos (eflorescencias) se compone prácticamente de sulfato de calcio (ver Figura 8), mientras que en áreas más internas del soporte pétreo se hallan, además, otras sales de calcio como, cloruros y nitratos (Osete, Kröner y Doménech, 2010, p. 4).

En rocas sedimentarias estratificadas, la humedad se traslada principalmente a través de las capas que son ricas en poros capilares. En la superficie de estas capas definidas es donde tiene lugar la cristalización de sales. Este proceso se ve incrementado por la acción del viento que va erosionando estas franjas al introducirse en las cavidades creadas y forma pequeños remolinos (Torraca, 2009, p. 87). El resultado es una superficie pétrea con estratificaciones definidas por la erosión y pulverulencia del material.

Este daño queda patente en varias zonas de los revestimientos internos de la acrópolis, sobre todo en las franjas bajas de los muros, donde se observa cómo se está formando esta estratificación y se produce la pulverulencia y desprendimiento del estuco superpuesto (ver Figura 9). En otros casos, el grado de estratificación está mucho más avanzado, razón por la que se perdió por completo el estuco. Este fenómeno ocurre de forma aislada solo en aquellos sillares realizados con piedra estratificada, por lo que en una misma superficie se pueden hallar zonas en buen estado, y zonas correspondientes al perímetro de un sillar determinado, afectadas por dicha patología (ver Figura 10).

A mediados del siglo XX, se comenzaron a emplear en estucos, pinturas y relieves del área maya los productos sintéticos que estaban teniendo resultados exitosos, principalmente resinas acrílicas (etil-metacrilato y resina acrílica en dispersión acuosa) y vinílicas (acetato de polivinilo). Estos productos, bajo condiciones ambientales estables como las de un museo, pueden resultar óptimos, pero en unas condiciones ambientales tan agresivas y cambiantes como las de los sitios arqueológicos del área no funcionaron, y en la década siguiente pudieron observarse resultados alarmantes que causaron daños irreversibles e incluso la pérdida de obras importantes. Por ello, es necesario apostar por soluciones alternativas más afines al medio y a los materiales que componen estos estucos, trabajando más del lado de la conservación con intervenciones mínimas, compatibles y revisables como principales criterios deontológicos.

Si los estucos mayas han sobrevivido hasta nuestros días, ¿qué mejor manera de intervenirlos que emplear sus propios materiales constitutivos? Intentar acercarse al producto que los expertos mayas elaboraron, mediante el empleo de los mismos recursos naturales y técnicas de manufactura aproximadas, puede ser una solución respetuosa para conservar estas obras sin causar efectos adversos en ellas. La compatibilidad de materiales en el campo de la conservación arqueológica resulta imprescindible para garantizar la estabilidad del comportamiento entre los materiales originales y los añadidos en la intervención. Se deben elegir materiales con una estructura y comportamiento similar a los originales, para que el nuevo conjunto se comporte adecuadamente en las condiciones previstas (Torraca, 1998, p. 9).

En el presente trabajo, se muestra el estudio comparativo entre dos morteros tradicionales, basados en las técnicas de manufactura maya y adicionados con tres exudados vegetales extraídos de cortezas de árboles oriundos. Se valora la resistencia que ambos presentan frente a los ciclos de cristalización de sales y la influencia que estos aditivos suponen en el incremento de la estabilidad, en relación con el tipo de árido con que se han dosificado. Este ensayo forma parte de un estudio más completo de caracterización, cuyo fin es la selección de aquellos morteros más adecuados con los trabajos de conservación y restauración realizados en La Blanca.

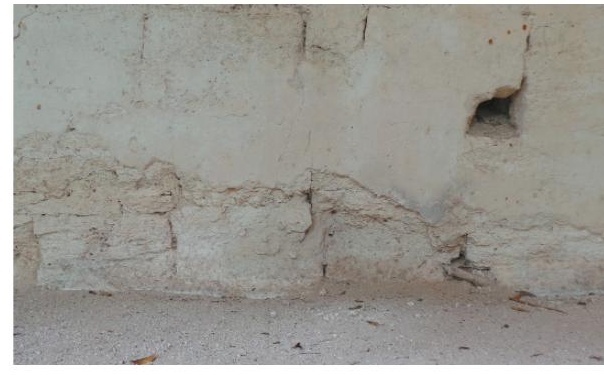

Figura 9. Desprendimiento del estuco y arenización del material pétreo por ciclos de cristalización salina. Archivo fotográfico de las autoras, 2018.

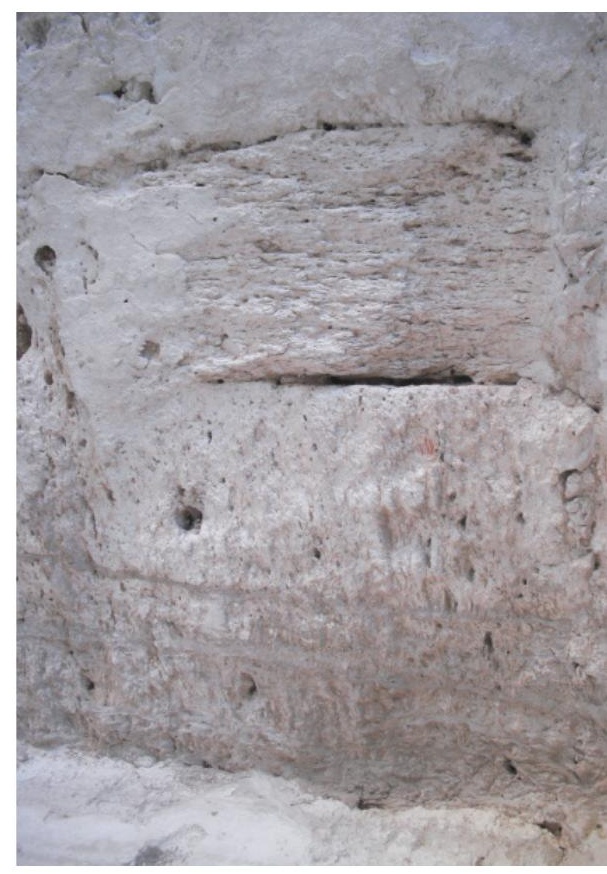

Figura 10. Estratificación de la piedra caliza que ha provocado el desprendimiento del estuco. Archivo fotográfico de las autoras, 2018. 
devenir Vol. 6, N¹2, JULIO - DICIEMBRE 2019, PP. 11-26 - Estudios I ISSN 2312-7562 | E-ISSN 2616-4949

UNIVERSIDAD NACIONAL DE INGENIERÍA, LIMA

doi: https://doi.org/10.21754/devenir.v6i12.741

\section{Objetivos}

Los objetivos específicos de este estudio son los siguientes:

- Evaluar la accesibilidad a los recursos naturales y la viabilidad de elaborar in situ morteros de cal basados en técnicas de manufactura maya para labores de conservación.

- Estudiar las diferencias físico-químicas que implica la formulación del mortero con dos áridos distintos bajo ensayo normalizado.

- Estudiar la influencia que tienen tres aditivos orgánicos en el incremento de la resistencia a la cristalización de sales, con respecto del mismo mortero sin aditivos.

- Determinar la viabilidad de emplear estos morteros como estratos de sacrificio en los paramentos que han perdido el estuco original por causa de los ciclos de cristalización de sales, así como en la reintegración de lagunas o refuerzos perimetrales para los estucos de La Blanca.

\section{Metodología}

Para la realización de este estudio, se han hecho distintas probetas de mortero en $\mathrm{Pe}$ tén, Guatemala, y se han trasladado a los laboratorios del Instituto Universitario de Restauración del Patrimonio, España, donde se ha curado, preparado y ensayado.

\section{Obtención de las materias primas}

Las materias primas esenciales que han sido seleccionadas y empleadas en la elaboración de las probetas estudiadas han sido las siguientes: cal apagada por pudrición, dos tipos de áridos locales, las cortezas de tres especies arbóreas originarias del área maya y agua.

- Cal: Se obtuvo cal viva de una calera local, ubicada en la población de San Benito, donde se calcina la piedra caliza en horno de piedra artesanal. Se adquirió en forma de terrón de cal viva, para proceder a su apagado por pudrición.

- Áridos: Los áridos propuestos para esta experimentación práctica se han seleccionado atendiendo a dos motivos principales: por su aparición reiterada en la bibliografía consultada, tanto etnográfica como etnohistórica, el sascab es el componente de carga empleado por los antiguos mayas como sustituto de la arena, con una composición calcárea y fina textura parecida a una «harina». En segundo lugar, se decidió introducir en el estudio un árido diferente del sascab por una cuestión de disponibilidad y facilidad, principalmente, ya que a menudo es difícil hallar sascaberas en las proximidades de un sitio arqueológico, por lo que se considera que se deben estudiar otros materiales, inmediatamente disponibles, para conocer su comportamiento y la idoneidad de ser empleados en labores de conservación, en caso de ser necesario (ver Figura 11).

Por ello, los áridos seleccionados fueron el sascab y la arena blanca presente en el sitio arqueológico La Blanca. El sascab se obtuvo de un afloramiento relativamente cercano, ubicado en la localidad de Paxcaman, Petén, a unos $50 \mathrm{~km}$ del sitio arqueológico. Para su preparación, ambos áridos fueron introducidos en depósitos de agua para eliminar, en la medida de lo posible, partículas orgánicas presentes que flotan hacia la superficie. Tras eliminar dichas partículas, el agua fue escurrida y el árido se dejó secar al sol. Una vez secos, se cribaron mediante un tamiz de $2 \mathrm{~mm}$ de luz para homogeneizar la granulometría y eliminar pequeñas piedras. El sascab presenta, generalmente, una granulometría menor que la malla del tamiz, por lo que en el cribado apenas se eliminaron partículas mayores. Por el contrario, la arena del sitio arqueológico presentaba

Figura 11. Sascabera en el sitio arqueológico Yaxhá, Petén. Archivo fotográfico de las autoras, 2018. una granulometría heterogénea con bastante cantidad de piedrecillas. 

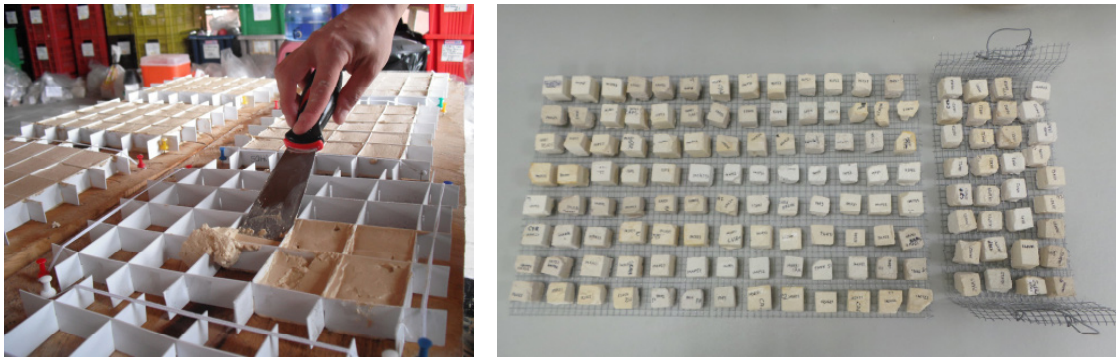

Figura 17. Moldeado de los morteros. Archivo fotográfico de las autoras, 2018.
Figura 18. Probetas colocadas sobre rejilla metálica. Archivo fotográfico de las autoras, 2018.

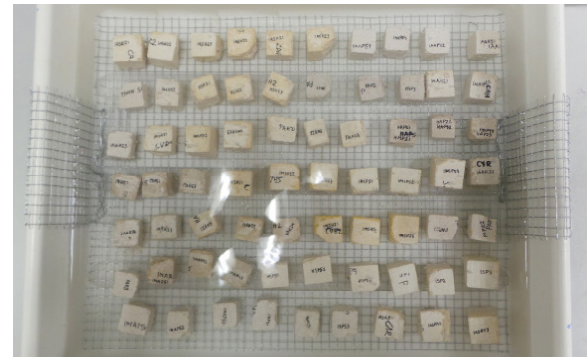

Figura 19. Baño en solución salina. Archivo fotográfico de las autoras, 2018.

Restauración del Patrimonio, España, donde se curaron durante un año en unas condiciones ambientales registradas de $23 \pm 5^{\circ} \mathrm{C}$ y $50 \pm 5 \% \mathrm{HR}$.

\section{Procedimiento de ensayo}

El ensayo se ha realizado según la normativa española UNE-EN 12370:1999 (CEN), Determinación de la resistencia a la cristalización de sales, que pretende evaluar la resistencia a los ciclos de cristalización de sales de las probetas de mortero endurecido para establecer comparativas entre ambos tipos de áridos, y determinar si la adición de exudados vegetales influye positiva o negativamente en su estabilidad. Aunque en principio está indicado para piedra natural de baja porosidad, se ha decidido adaptarlo al material en estudio, dado que la cristalización de sales in situ es un grave problema que afecta tanto a los estucos originales como a los de reposición, así como al soporte pétreo.

Después de secar hasta masa constante (ver Figura 18), las probetas son sumergidas en una solución de sulfato sódico decahidratado al $14 \%$ durante 2 horas, a una temperatura de $20^{\circ} \mathrm{C}$. Transcurrido ese tiempo, se introducen en una estufa de aire caliente a $60^{\circ} \mathrm{C}$ durante 15 horas y se dejan enfriar durante 2 horas más antes de volver a introducirlas en el baño de sales (ver Figura 19). Siguiendo dicha normativa (UNE-EN 12370, 1999), este ciclo se repite 15 veces, excepto si las probetas se rompen antes. Tras el último ciclo, las probetas se neutralizan en agua durante 24 horas a $23 \pm 5^{\circ} \mathrm{C}$ y se secan hasta masa constante. Los resultados se expresan como la diferencia de masa relativa $\Delta m$ (pérdida o ganancia de masa) con respecto de la masa seca inicial $M d$, mediante la fórmula:

$$
\Delta \mathrm{m}=(\mathrm{mf}-\mathrm{md} 1) / \mathrm{md} \cdot 100
$$

O como el número de ciclos necesarios para degradar las probetas.

\section{Resultados}

Como se observa en la Figura 20, los resultados que se han obtenido con este ensayo muestran dos comportamientos muy diferenciados. La resistencia a la degradación es significativamente mayor en el grupo dosificado con sascab (ver Figura 21). En este grupo, todos los tipos de mortero muestran un promedio de ciclos resistidos bastante alto, que se sitúa entre 6 y 11,67 ciclos. Por otro lado, el grupo realizado con arena blanca del sitio presenta un promedio de ciclos resistidos en torno a 4 , independientemente del tipo de exudado y el porcentaje de maceración que contengan.

La arena blanca obtenida del sitio arqueológico presenta una granulometría heterogénea y de hasta $2 \mathrm{~mm}$ de tamaño de partícula, que podría ser el motivo de la disminución de resistencia mecánico-química, ya que, cuando los áridos son pobres en partículas finas, el mortero se comporta irregularmente y el conglomerante actúa como carga fina en vez de como cementante (Alonso Olvera, 2013, p. 82). El sascab, por el contrario, presenta una granulometría bastante más pequeña y regular. Esta carac- 
devenir Vol. 6, N¹2, JULIO - DICIEMBRE 2019, PP. 11-26 - Estudios I ISSN 2312-7562 | E-ISSN 2616-4949 UNIVERSIDAD NACIONAL DE INGENIERÍA, LIMA

doi: https://doi.org/10.21754/devenir.v6i12.741

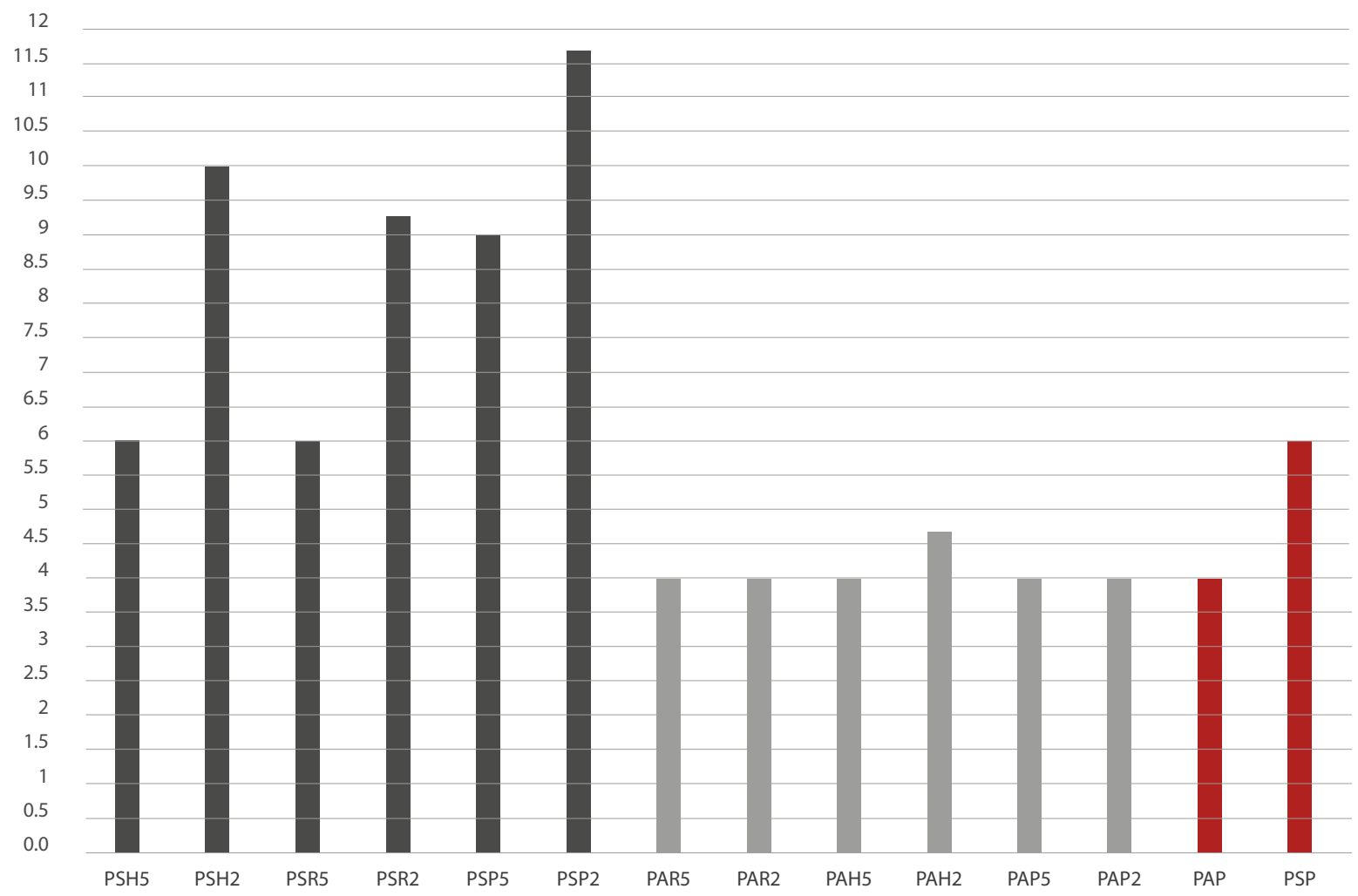

Figura 20. Número de ciclos soportados por cada tipo de mortero. Elaboracion propia, 2018.

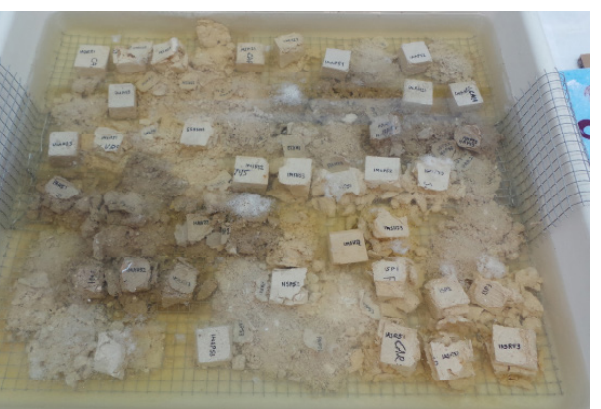

Figura 21. Desintegración de gran parte de las probetas en el baño 5 . Archivo fotográfico de las autoras, 2018. terística podría ser negativa, ya que el exceso de arenas finas también produce una disminución en la resistencia mecánica, reducción de la permeabilidad y fomento de la formación de grietas y fisuras al endurecer el mortero (Alonso Olvera, 2013, p. 82). Pero, en este caso, la estructura más densa, compacta y con menor porosidad ha contribuido a un comportamiento hídrico menor, absorbiendo menos cantidad de agua y, por tanto, introduciendo menor cantidad de solución salina a la red porosa. A estos factores, se suma otro claramente influyente en el resultado.

En la Tabla 2, se observa que el mortero testigo del grupo realizado con sascab (PSP), muestra una disminución en la resistencia, puesto que este grupo cuenta con un promedio de ciclos de 6 , mientras que el promedio de ciclos soportados aumenta en los morteros que contienen aditivo orgánico en casi el doble $(11,67)$.

Otro dato que muestra la influencia de los aditivos en la mejora de la resistencia es la diferencia entre las concentraciones añadidas. Los morteros PSH5, PSR5 y PSP5, que contienen el aditivo en proporción 0,5 \%, han soportado menor número de ciclos, al ser su comportamiento más similar al del mortero patrón, realizado sin aditivo. Por otra parte, sus homólogos adicionados con el $2 \%$ de concentración muestran los rangos más elevados. Desde el punto de vista comparativo, entre el tipo de exudado añadido también se observa una diferencia clara. Los morteros realizados con maceración de habin y de ramón muestran resistencias similares, siendo de 6 en concentración de 0,5\% a 10,33 en concentración de $2 \%$ para el adicionado con habin y de $6(0,5 \%)$ a 9,33 (2\%) para el adicionado con ramón. Los morteros realizados con pixoy muestran resistencias superiores en ambas concentraciones, siendo de $9(0,5 \%)$ y 11,67 (2\%), de lo que se deduce que este exudado proporciona un mortero más compacto, fuerte y con menor porosidad abierta al endurecer. 
Tabla 2. Masa inicial, masa final, número de ciclos soportados y pérdida de masa final de cada tipo de mortero.

\begin{tabular}{|c|c|c|c|c|}
\hline Probeta & $\operatorname{Md}\left({ }^{\delta}\right)$ & Mf $\left(^{\delta}\right)$ & $\mathrm{N}^{\circ}$ Ciclos $\left({ }^{\delta}\right)$ & $\%$ Pérdida $\left({ }^{\delta}\right)$ \\
\hline PSH5 & $10,29(0,6)$ & - & $6(0,0)$ & -100 \\
\hline PSH2 & $9,46(0,1)$ & - & $10,33(3,4)$ & -100 \\
\hline PSR5 & $10,83(1,3)$ & - & $6(0,0)$ & -100 \\
\hline PSR2 & $9,72(0,3)$ & - & $9,33(4,6)$ & -100 \\
\hline PSP5 & $10,94(0,3)$ & - & $9,00(5,2)$ & -100 \\
\hline PSP2 & $10,42(0,5)$ & - & $11,67(5,0)$ & -100 \\
\hline PAR5 & $8,96(0,2)$ & - & $4(0,0)$ & -100 \\
\hline PAR2 & $7,46(0,0)$ & - & $4(0,0)$ & -100 \\
\hline PAH5 & $8,22(0,3)$ & - & $4(0,0)$ & -100 \\
\hline PAH2 & $8,26(1,7)$ & - & $4,67(1,1)$ & -100 \\
\hline PAP5 & $6,93(0,7)$ & - & $4(0,0)$ & -100 \\
\hline PAP2 & $6 ., 71(0,5)$ & - & $4(0,0)$ & -100 \\
\hline PAP & $5,95(0,7)$ & - & $4(0,0)$ & -100 \\
\hline PSP & $10,42(0,5)$ & - & $6(0,0)$ & -100 \\
\hline
\end{tabular}

En el caso del grupo elaborado con arena blanca, como se ha comentado, las resistencias son menores en general, no observándose apenas diferencia entre los morteros adicionados con uno u otro exudado, ni con el menor o el mayor porcentaje de concentración. Todos ellos tienen una resistencia de 4 ciclos de cristalización y se desintegran (la mayoría de probetas, menos en el caso del habin al $2 \%$, que tiene un promedio de 4,67) durante la inmersión del quinto baño en solución salina.

Por su parte, el mortero patrón, realizado sin aditivo, revela el mismo resultado (4 ciclos), por lo que la adición de las gomas vegetales en este grupo realizado con arena blanca no parece influir en la mejora de la resistencia a la cristalización de sales.

\section{Conclusiones}

La cal apagada por exposición al aire libre, o por pudrición como se conoce en el área maya, es uno de los métodos de apagado presente en la tradición maya para la elaboración de sus estucos. Dada la elevada humedad del área, este sistema supone un método fácil y rápido de apagado, en el que el óxido de calcio resultante de la quema de piedra caliza se transformaba en hidróxido de calcio por la combinación con partículas de agua. A diferencia del apagado por fusión, mediante este procedimiento la cal viva no requería ser transportada a otra zona (con el riesgo que conlleva, dado su poder cáustico), ni transportar grandes cantidades de agua hasta el área de la quema de cal, 
generalmente lejos de la zona de construcción. Este método todavía se emplea hoy en día en Petén y pueden verse, en las pequeñas caleras locales, extensiones de cal expuesta que se va removiendo para que las piedras se vayan hinchando y se conviertan en pequeñas «migas», sinónimo de su hidratación. Por ser este uno de los posibles sistemas de apagado que los mayas emplearon para elaborar sus estucos, se considera importante su caracterización y evaluación frente a determinados ensayos físicos, químicos y mecánicos para confirmar la viabilidad de ser empleados como morteros de reposición en las labores de conservación y restauración del proyecto. En este trabajo, se han expuesto los resultados del ensayo de cristalización de las sales, mecanismo de deterioro que afecta acusadamente en los estucos originales de La Blanca, y de la gran mayoría de sitios del área maya, una vez que se han liberado y sacado a la luz las estancias donde se ubican.

Tras evaluar los resultados, se concluye, en primer lugar, que la incorporación en el mortero de un árido u otro (sascab o arena blanca) condiciona considerablemente la resistencia frente a este agresivo agente de deterioro. El número de ciclos completos, necesarios para desintegrar el mortero, son muy superiores en el grupo elaborado con sascab que en el que contiene arena blanca. Dado que químicamente ambos áridos están compuestos principalmente por calcita, se deduce que la diferencia de comportamiento radica en la forma y tamaño de sus partículas y en cómo estas interactúan con el conglomerante y con los aditivos orgánicos para formar una estructura más o menos compacta y con mayor o menor porosidad.

En la Tabla 3, se muestran otros datos obtenidos en estudios previos que forman parte de esta tesis. La absorción de agua por capilaridad es superior en los morteros realizados con arena blanca, con coeficientes entre 0,73 y $1,09 \mathrm{gr} / \mathrm{cm} 2 . \mathrm{s} 1 / 2$, mientras que el grupo realizado con sascab presenta coeficientes de 0,44 a $0,49 \mathrm{gr} / \mathrm{cm} 2 . \mathrm{s} 1 / 2$, por lo que la penetración de la solución salina a la red porosa del mortero es menor en este grupo. Esto contribuye a que se prolongue su resistencia, pues soporta un mayor número de ciclos. Por otra parte, se encontraron diferencias significativas entre la densidad aparente de cada grupo de morteros, siendo de entre 1401,51 y $1822,37 \mathrm{Dap} \mathrm{kg} / \mathrm{m} 3$ para el grupo elaborado con sascab, y de 1223,24 y 1286,33 Dap kg/m3 para el grupo elaborado con arena blanca (ver Tabla 3), lo que revela la presencia de más espacios de aire en su interior. Estos datos evidencian que la porosidad de este último grupo es bastante superior al sascab, al formar un mortero menos compacto, con menor resistencia mecánica y con mayor accesibilidad del agua, por lo que resulta menos adecuado para ser empleado como estrato de sacrificio en las labores de conservación del sitio.

Con la aplicación de estos estratos, se pretende mitigar los efectos de la cristalización sobre la piedra caliza que da soporte al estuco y que ya ha causado la desintegración de algunas áreas de estuco, siempre en las franjas bajas de los paramentos. Con la piedra al descubierto, las sales solubles, presentes en el agua que asciende por capilaridad desde el subsuelo, se cristalizan reiteradamente sobre la superficie de la piedra, de manera que avanzan su deterioro y desintegración, y desencadenan daños en las zonas circundantes. Justamente, para este cometido, se requiere un mortero resistente a este agente dañino que sufra los efectos de la cristalización para evitar que se dañe el soporte pétreo.

Cabe decir que los morteros que aquí se han estudiado forman parte de otros seis grupos técnicos en los que se ha empleado distinta metodología de apagado de la cal y se han sometido todos ellos a distintos ensayos de caracterización física, mecánica y química, con la intención de seleccionar aquel que reúna las mejores cualidades para el propósito.

\section{Referencias}

Alonso Olvera, A. (2013). Las propiedades de la cal y la manufactura de materiales de cal. En L. Barba y I. Villaseñor (Eds.), La cal. Historia, propiedades y usos (pp. 73-94). México: Universidad Nacional Autónoma de México. 
Tabla 3. Coeficiente de absorción de agua por capilaridad y densidad aparente en seco.

\begin{tabular}{|c|c|c|c|}
\hline Probeta & $\mathrm{gr} / \mathrm{cm} 2 . \mathrm{s} 1 / 2$ & Dap Kg/m3 & $N^{\circ}$ Ciclos $\left(^{\delta}\right)$ \\
\hline PSR2 & 0,49 & 1401,51 & $9,33(4,6)$ \\
\hline $\mathrm{PSH} 2$ & 0,45 & 1432,76 & $10,33(3,4)$ \\
\hline PSP2 & 0,47 & 1475,06 & $11,67(5,0)$ \\
\hline PSH5 & 0,47 & 1456,90 & $6(0,0)$ \\
\hline PSR5 & 0,44 & 1590,00 & $6(0,0)$ \\
\hline PSP5 & 0,45 & 1822,37 & $9.00(5,2)$ \\
\hline PAH2 & 0,91 & 1278,09 & $4.67(1,1)$ \\
\hline PAP2 & 1,09 & 1223,24 & $4(0,0)$ \\
\hline PAR2 & 0,9 & 1256,82 & $4(0,0)$ \\
\hline PAH5 & 1,04 & 1267,43 & $4(0,0)$ \\
\hline PAP5 & 0,83 & 1286,33 & $4(0,0)$ \\
\hline PAR5 & 0,73 & 1291,94 & $4(0,0)$ \\
\hline PAP & 0,85 & 1284,47 & $4(0,0)$ \\
\hline PSP & 0,45 & 1479,69 & $6(0,0)$ \\
\hline
\end{tabular}

Barba, L. (2013). El uso de la cal en el mundo mesoamericano prehispánico. En L. Barba y I. ViIlaseñor (Eds.), La cal. Historia, propiedades y usos, (pp. 19-46). México: Universidad Nacional Autónoma de México.

De Landa, D. (2002). Relación de las cosas de Yucatán. Madrid, España: Dastin.

Doehne, E. y Price, C. (2010). Stone conservation. An overview of current Research. EE. UU.: The Getty Conservation Institute.

Grübe, N. (2000). Los mayas. Una civilización milenaria. Könemann, Colonia.

Hall, C., Hoff, W. D. y Hamilton, A. (2008). Capillary rise dynamics in masonry structures: The role of wáter flux in long-term damage. En Lukaszewicz, J. W y Niemcewicz, P. (Eds.), Proceedings of the 11th International Congress on Deterioration and Conservation of Stone (pp. 15-20). Torurí, Poland: Nicolaus Copernicus University.

Kröner, S., Mas i Barberà, X., Osete, L. y Guash, N. (2010). Identificación y caracterización de materiales pétreos en patrimonio histórico-artístico. Universitat Politècnica de València.

Lewin, S. Z. (1982). The mechanism of masonry decay through crystallization. En Conservation of Historic Stone Buildings and Monuments: Report of the Committee on Conservation of Historic Stone Buildings and Monuments, National Materials Advisory Board, Commission on Engineering and Technical Systems, National Research Council (pp. 120-44). Washington, D. C.: National Academy Press.

Littman, E. (1960). Ancient Mesoamerican Mortars, Plasters and Stuccos: The Use of Bark Extracts in Lime Plasters. American Antiquity, 25(4), 593-597. 
devenir Vol. 6, N¹2, JULIO - DICIEMBRE 2019, PP. 11-26 - Estudios I ISSN 2312-7562 | E-ISSN 2616-4949

UNIVERSIDAD NACIONAL DE INGENIERÍA, LIMA

doi: https://doi.org/10.21754/devenir.v6i12.741

Lorenzo, F. y Carrascosa, B. (2014). La restauración de los estucos incisos de La Blanca. Estudios previos en morteros de cal con aditivos vegetales y evaluación de su comportamiento en clima tropical. En Emerge. Jornadas de investigación emergente en conservación y restauración de patrimonio (pp. 63-70). Universitat Politècnica de València.

Lorenzo, F. y Carrascosa, B. (2017). Control in situ de la permeabilidad de agua en morteros de cal con aditivos orgánicos empleados en la reintegración de estucos mayas. Arché, (11-12), 11-18.

Ministerio de Agricultura, Ganadería y Alimentación. (2001). Mapa fisiográfico geomorfológico de la República de Guatemala a escala 1: 250,00. Memoria técnica. Unidad de políticas e información estratégica (UPIED-MAGA). Programa de emergencias por desastres naturales. Ministerio de agricultura, ganadería y alimentación, Guatemala.

Magaloni, D. (1995). El espacio pictórico Teotihuacano. Tradición y técnica. En B. de la Fuente y L. Staines (Eds.), La pintura mural prehispánica en México (pp. 187-226). Instituto de Investigaciones Estéticas, Universidad Nacional Autónoma de México.

Magaloni, D. (2001). Materiales y técnicas de la pintura mural maya. En B. de la Fuente (Dir.) y L Staines (Coords.), La pintura mural prehispánica en México II. Área maya. Tomo III. (pp. 155-196). México: Instituto de Investigaciones Estéticas Universidad Nacional Autónoma de México.

Mas-Guindal A.J., Martín F., Nuere E. y García de Miguel, M. a. (1999). Procedimientos y técnicas constructivas del Patrimonio. Madrid, España: Munilla-Lería.

Moreno, H., Ibáñez, S. y Gisbert, J. M. (s. f.). Rocas sedimentarias químicas. Universitat Politècnica de València.

Muñoz Cosme, G. (2006). Introducción a la arquitectura maya. Valencia, España: Biblioteca TC.

Osete, L., Kröner, S. y Doménech, M. T. (2007). Informe analítico. Instituto Universitario de Restauración del Patrimonio, Universitat Politècnica de Valencia.

Schreiner, T. (2002). Traditional Maya lime production: Environmental and Cultural implications of a Native American Technology [Tesis doctoral inédita presentada]. University of California, Berkley.

Torraca, G. (1998). I materiali e il restauro. Il ritorno de la calce. Le dimore Storiche, (14), 9-10.

Torraca, G. (2009). Lectures on materials science for architectural conservation. EE. UU.: The Getty Conservation Institute.

UNE-EN 12370. (marzo, 1999). Determinación de la resistencia a la cristalización de sales. @Aenor.

Villaseñor, I. y Barba, L. (2011). El estudio de materiales constructivos en la arqueología mesoamericana. En Anales de Antropología 45 (pp. 79-98). Universidad Nacional Autónoma de México.

Villaseñor, I. y Barba, L. (2012). Los orígenes tecnológicos de la cal. Cuicuilco, 19(55), 11-41.

Villaseñor, I. y Graham, E. (2010). The use of volcanic materials for the manufacture of pozzolanic plasters: A preliminary report. Journal of Archaeological Science, (37), 1339-1347. 